\title{
Crystal structure of tetrapotassium diarsenidomercurate(II), $\mathbf{K}_{4}\left[\mathrm{HgAs}_{2}\right]$
}

\author{
M. Asbrand, B. Eisenmann
}

Technische Hochschule Darmstadt, Eduard-Zintl-Institut, Anorganische Chemie II, Hochschulstr. 10, D-64289 Darmstadt, Germany

and M. Somer

Max-Planck-Institut für Festkörperforschung, Heisenbergstr. 1, D-70569 Stuttgart, Germany

Received July 30, 1996, transferred to 2nd update of database ICSD in 1997, CSD-No. 402573

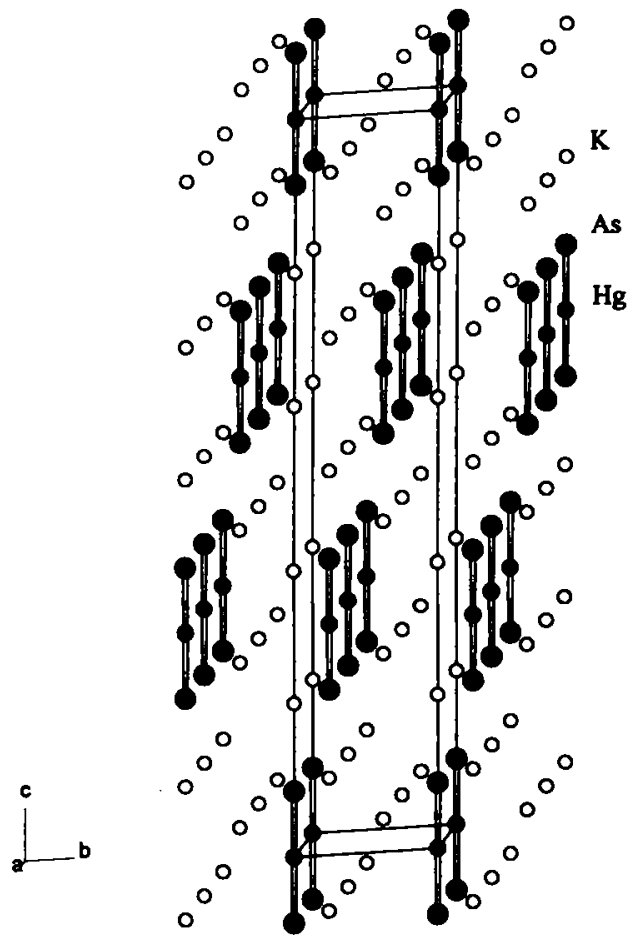

As2 $\mathrm{HgK}_{4}$, trigonal, $R \overline{3} m$ (No. 166), $a=5.763(1) \AA$, $c=27.696(2) \AA, V=796.6 \AA^{3}, Z=3, R(F)=0.040, R_{w}\left(F^{2}\right)=0.099$.

Source of material: $\mathrm{K}_{4}\left[\mathrm{HgAs}_{2}\right]$ was synthesized from stoichiometric amounts of the elements in sealed niobium ampoules. The samples were heated to $950 \mathrm{~K}$ within $4 \mathrm{~h}$ and cooled to room temperature for $40 \mathrm{~h}$. An excess of potassium (10-20 at\%) was used to improve the crystallization and removed by vacuum distillation.

$\mathrm{K}_{4}\left[\mathrm{HgAs}_{2}\right]$ crystallizes in the $\mathrm{Na}_{4}\left[\mathrm{HgP}_{2}\right]$ structure type (see ref. 1) containing isolated linear $[\mathrm{As}-\mathrm{Hg}-\mathrm{As}]^{4-}$ anions which are isosteric to the $\mathrm{HgCl}_{2}$ molecule. The bond length $d(\mathrm{Hg}-\mathrm{As})=$ $2.463 \AA$ is in good agreement with the sum of covalent radii for single bonds (see refs. 2,3). The anion belongs to the isoelectronic series $\mathrm{HgCl}_{2},\left[\mathrm{CuBr}_{2}\right]^{-},\left[\mathrm{HgO}_{2}\right]^{2-},\left[\mathrm{AgO}_{2}\right]^{3-},\left[\mathrm{HgP}_{2}\right]^{4-},\left[\mathrm{CuSb}_{2}\right]^{5-}$ (see refs. $4,5,6$ ).

Table 1. Parameters used for the $\mathrm{X}$-ray data collection

\begin{tabular}{|c|c|}
\hline $\begin{array}{l}\text { Crystal: } \\
\text { Wavelength: } \\
\mu:\end{array}$ & $\begin{array}{l}\text { black prism, size } 0.1 \times 0.1 \times 0.3 \mathrm{~mm} \\
\text { Mo } K_{\alpha} \text { radiation }(0.71073 \AA) \\
221.59 \mathrm{~cm}^{-1}\end{array}$ \\
\hline $\begin{array}{l}\mu: \\
\text { Diffractometer: }\end{array}$ & Philips PW1100 \\
\hline Scan mode: & $\theta / 2 \theta$ \\
\hline $\mathrm{T}_{\text {measurement: }}$ & $293 \mathrm{~K}$ \\
\hline $2 \theta_{\max }:$ & $60^{\circ}$ \\
\hline $\mathbf{N}(h k l)_{\text {wnique: }}$ & 329 \\
\hline Criterion for $I_{0}:$ & $I_{0}>2 \sigma\left(I_{0}\right)$ \\
\hline$N(\text { param })_{\text {refined. }}$ & 12 \\
\hline Program: & SHELXL-93 \\
\hline
\end{tabular}

Table 2. Final atomic coordinates and displacement parameters (in $\AA^{2}$ )

\begin{tabular}{lllllllllll}
\hline Atom & Site & $x$ & $y$ & $z$ & $U_{11}$ & $U_{22}$ & $U_{33}$ & $U_{12}$ & $U_{13}$ & $U_{23}$ \\
\hline $\mathrm{K}(1)$ & $6 c$ & 0 & 0 & $0.2083(1)$ & $0.027(1)$ & $U_{11}$ & $0.033(2)$ & $U_{11} / 2$ & 0 & 0 \\
$\mathrm{~K}(2)$ & $6 c$ & 0 & 0 & $0.3879(2)$ & $0.022(1)$ & $U_{11}$ & $0.062(2)$ & $U_{11} / 2$ & 0 & 0 \\
$\mathrm{Hg}$ & $3 a$ & 0 & 0 & 0 & $0.0169(3)$ & $U_{11}$ & $0.0243(4)$ & $U_{11} / 2$ & 0 & 0 \\
As & $6 c$ & 0 & 0 & $0.08893(5)$ & $0.0192(5)$ & $U_{11}$ & $0.0244(7)$ & $U_{11} / 2$ & 0 & 0 \\
\hline
\end{tabular}

\section{References}

1. Eisenmann, B.; Somer, $\mathbf{M}$.: Intermetallische Verbindungen mit $\mathrm{HgCl}_{2}$-isosteren Anionen: Strukturelle und Schwingungsspektroskopische Untersuchungen von $\mathrm{Na}_{4} \mathrm{HgP}_{2}, \mathrm{~K}_{4} \mathrm{ZnP}_{2}, \mathrm{~K}_{4} \mathrm{CdP}_{2}$ und $\mathrm{K}_{4} \mathrm{HgP}_{2}$. Z. Naturforsch. 44b (1989) 1228-1232.

2. Pyykkö, P.: Relativistic Effects in Structural Chemistry. Chem. Rev. 88 (1988) 563-594

3. Pauling, L.: Die Natur der Chemischen Bindung. Verlag Chemie, Weinheim 1968.
4. Savelsberg, G.; Schäfer, H.: K5CuSb2 - eine Verbindung mit gestreckten Sb-Cu-Sb Einheiten. Z. Naturforsch. 34b (1979) 771-773.

5. Eisenmann, B.; Klein, J.; Somer M.: Crystal structure of tetrapotassium diarsenidocadmate, K4CdAs2. Z. Kristallogr. 197 (1991) 271-272.

6. Eisenmann, B.; Klein, J.; Somer, M.: Linear anions $\left[\mathrm{CuAs}_{2}\right]^{5-}$, $\left[\mathrm{AuP}_{2}\right]^{5-}$ and [AuAs$\left._{2}\right]^{5-}$ in potassium compounds. J. Alloys Comp. 178 (1992) 431-439.

7. Sheldrick, G. M.: SHELXL-93, a program for refining crystal structures. University of Göttingen, Germany 1993. 